\title{
The Energy Spectrum of Reactor Antineutrinos
}

\author{
Daniel Dwyer* \\ Lawrence Berkeley National Laboratory, Berkeley, CA, USA \\ E-mail: dadwyeralbl.gov
}

\begin{abstract}
Measurements of electron antineutrinos emitted by nuclear reactors have been central to our understanding the nature of these particles. These successes have been clouded by disagreements between existing models and measurements of the rate and energy spectra of $\bar{v}_{e}$ from reactors. Direct calculation of energy spectra based on nuclear fission and decay databases, despite significant uncertainties, suggests a possible explanation for the observed spectral shape. Here I discuss the details of these comparisons of models with measurements, their implications, and possible future measurements with potential to clarify these outstanding issues.
\end{abstract}

XVI International Workshop on Neutrino Telescopes,

2-6 March 2015

Palazzo Franchetti, Istituto Veneto, Venice, Italy

\footnotetext{
* Speaker.
} 


\section{Introduction}

Antineutrinos emitted by nuclear reactors have been an effective tool for experiments in particle physics. Reactors are the most intense man-made neutrino source, emitting approximately $10^{21} \bar{v}_{e}$ per second from a standard $3 \mathrm{GW}_{\text {th }}$ reactor. Utilizing this intense flux, the first neutrino interactions were observed in the late 1950s [1]. Subsequent measurements of reactor $\bar{v}_{e}$ have provided the most distinct signal of the oscillation of neutrino flavor [2, 3], demonstrating violation of lepton flavor conservation and implying that neutrinos are massive particles. More recently, precision measurements of reactor $\bar{v}_{e}$ disappearance over $2 \mathrm{~km}$ distances have demonstrated significant flavor oscillation driven by the larger separation to the third neutrino mass eigenstate: $m_{3}^{2}-m_{1}^{2}=\Delta m_{31}^{2} \approx \Delta m_{32}^{2}[4,5,6]$. All together, reactor $\bar{v}_{e}$ have provided measurements of four of the six parameters describing neutrino mass and mixing: $\left|\Delta m_{21}^{2}\right|,\left|\Delta m_{31}^{2}\right|, \theta_{12}$, and $\theta_{13}$. Upcoming experiments intend to use reactors to elucidate the ordering of the neutrino mass eigenstates (i.e. $m_{3}>m_{1}, m_{2}$ or $\left.m_{3}<m_{1}, m_{2}\right)[7,8]$.

Despite these successes, the standard predictions of the $\bar{v}_{e}$ flux from reactors disagree with precise measurements. Accounting for detailed corrections to the estimation of $\bar{v}_{e}$ flux give a predicted interaction rate $6 \%$ higher than measurements [9]. This discrepancy has come to be known as the reactor antineutrino rate anomaly, and has been considered as possible evidence for oscillation to a hypothetical non-interacting (i.e. sterile) fourth neutrino flavor. To complicate matters further, recent precise measurements of reactor $\bar{v}_{e}$ energy spectra show a significant deviation from standard predictions, particularly in the energy range of 5 to $7 \mathrm{MeV}[4,5,6]$.

In the following proceedings, I review the models used to predict reactor $\bar{v}_{e}$ emission and describe the limitations of these predictions. I then discuss the comparison between the models and measurements, and some unexpected observations which arise. This is followed by a brief discussion of additional developments in the study of $\bar{v}_{e}$ emission, and suggestions concerning future experiments which could help resolve to these questions.

\section{Modeling reactor $\bar{v}_{e}$ emission}

The physical process behind the emission of antineutrinos from nuclear reactors is relatively clear. Fission of actinides in nuclear reactors, primarily ${ }^{235} \mathrm{U},{ }^{238} \mathrm{U},{ }^{239} \mathrm{Pu}$, and ${ }^{241} \mathrm{Pu}$, produce neutron-rich daughter nuclides. Each unstable daughter nuclide undergoes successive beta decays until it is converted to a stable, or at least long-lived, nuclide. An average of six beta decays occur for each initial actinide fission. Each beta decay emits an electron and a corresponding electron antineutrino. Therefore, the total antineutrino flux from a reactor $S_{t o t}$ can be expressed as,

$$
S_{\text {tot }}\left(E_{\bar{V}}\right)=\sum_{i} R_{i} S_{i}\left(E_{\bar{V}}\right)
$$

Here $R_{i}$ is the decay rate of the $i^{\prime}$ th daughter nuclide, which could equivalently be identified by its unique proton number, neutron number, and possible excited meta-stable isomeric level $(Z, N$, $m)$. The corresponding antineutrino spectrum emitted per decay of this nuclide is given by $S_{i}$. Each daughter nuclide can generally beta decay to one of a number of possible energy levels of the subsequent nuclide. The average antineutrino spectra for a particular nuclide is therefore a sum of 
spectra $S_{i j}$,

$$
S_{i}\left(E_{\bar{V}}\right)=\sum_{j} f_{j} S_{i j}\left(E_{\bar{V}}\right)
$$

where $f_{j}$ is relative probability of a decay to the $j$ 'th energy level of the subsequent nuclide.

While such summation calculations of the antineutrino spectrum $S_{t o t}$ from a reactor are simple in principle, our limited knowledge of nuclear physics render these difficult in practice. According to current nuclear databases, $>1000$ fission daughter nuclides with a total of $\sim 10,000$ unique beta decay transition contribute to the total $\bar{v}_{e}$ spectrum $[10,11]$. Under equilibrium conditions, the decay rates of these nuclides in the reactor, $R_{i}$, can be estimated from the cumulative fission yields for these nuclides. Fission yields tabulated by the ENDF [12, 13, 14] and JEFF [15] nuclear databases differ by an amount which is greater than their claimed uncertainties, resulting in $\bar{v}_{e}$ spectra inconsistent at the $\sim 10 \%$ level according to a recent study [16]. The nuclear energy levels and decay structures are poorly known for most of these fission daughters, resulting in large uncertainties in the relative decay probabilities $f_{j}$. There are also concerns about the existence of unknown energy levels beyond those tabulated by current nuclear databases. Lastly, uncertainties in nuclear corrections to the $\bar{v}_{e}$ beta decay energy spectra $S_{i j}$ add additional, if smaller, uncertainty [17]. Combined, these poorly-quantified systematic effects introduce uncertainties of approximately $10 \%$ to $15 \%$ to calculations of the total $\bar{v}_{e}$ rate and spectrum.

For this reason, physicists have generally relied on an alternate method to estimate the $\bar{v}_{e}$ spectrum from reactors. In this $\beta$-conversion method, the cumulative spectra of electrons emitted during active fission of actinide samples are used to estimate the corresponding $\bar{v}_{e}$ spectra. Benchmark electron spectral measurements for fission of ${ }^{235} \mathrm{U},{ }^{239} \mathrm{Pu}$, and ${ }^{241} \mathrm{Pu}$ were carried out at the ILL reactor using the BILL spectrometer in the 1980's [18, 19, 20]. More recently, a measurement of ${ }^{238} \mathrm{U}$ fission was also completed [21]. To first order, the electron and $\bar{v}_{e}$ energy spectra are strongly correlated due to the kinematic relationship between these two particles emitted during beta decay. The primary uncertainties arise from nuclear corrections which can introduce a slight asymmetry between the energy spectra of these two particles. A hybrid method used tabulated nuclear data in order to reduce these uncertainties [22]. Combined with other improvements in the calculation, this hybrid model predicted a rate $6 \%$ higher than previous calculations and claimed an uncertainty in the model of roughly $\sim 2 \%$, which was consistent with an alternate study [23]. Fig. 1 compares summation and $\beta$-conversion predictions of the $\bar{v}_{e}$ spectrum emitted by a nominal nuclear reactor.

\section{Comparison with measurements}

While the $\beta$-conversion method provides a more precise prediction for the $\bar{v}_{e}$ spectrum from reactors, it is unexpectedly inconsistent with actual measurements. First, it predicts a total $\bar{v}_{e}$ interaction rate which is $6 \%$ higher than the global average measurement. This rate discrepancy has been considered possible evidence for the oscillation of neutrinos to a hypothetical sterile flavor [9].

More recently, experiments aiming to measure the mixing angle $\theta_{13}$ have made precise measurements of reactor $\bar{v}_{e}$ energy spectra $[6,24,25]$. All three experiments show a common but unexpected deviation of the energy spectrum relative to $\beta$-conversion predictions, with particularly significant tension for $\bar{v}_{e}$ with energies from 5-7 MeV. This feature has come to be known as the 


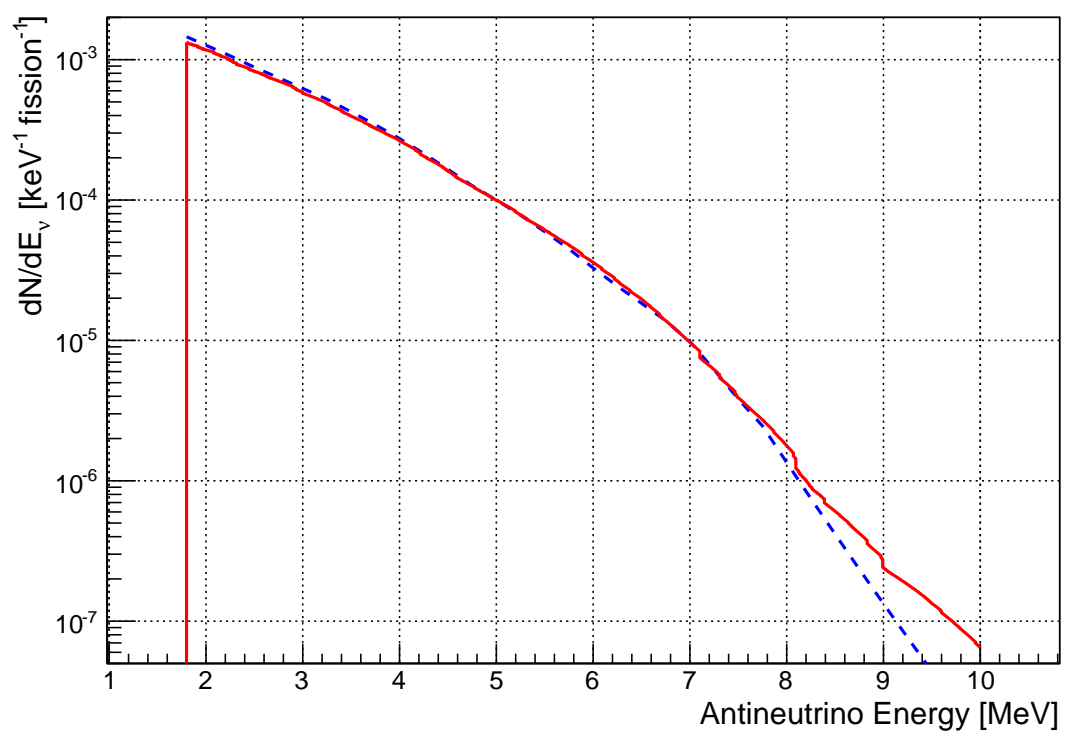

Figure 1: The energy spectrum of electron antineutrinos emitted from a nominal pressurized water reactor calculated using the summation method with the ENDF/B.VII.i nuclear database (solid red line [10]). The spectrum is calculated between $1.8 \mathrm{MeV}$, the threshold for $\bar{v}_{e}$ interaction via inverse beta decay, and $10 \mathrm{MeV}$. Calculation via the $\beta$-conversion method predicts a slightly steeper spectrum (blue dashed line [23]). The deviation between the two models above $7.5 \mathrm{MeV}$ is attributed to systematic overestimation of the relative probability of high-energy beta decays in tabulated nuclear databases, also known as the Pandemonium effect [26].

reactor bump, or more accurately the reactor shoulder. This localized spectral feature does not seem compatible with the sterile neutrino hypothesis.

A drawback of the $\beta$-conversion method is that it provides few routes to explore possible origins of this spectral discrepancy. Given that the electron measurements combine the energy spectra from thousands of unique beta decay transitions, one cannot disentangle the contributions from individual nuclides. Although less precise, here the summation method can possibly provide guidance, by predicting the composition of decay transitions in the shoulder region. A small number of beta decay transitions would need to be exceptionally prominent in the summation in order to generate a localized spectral shoulder as shown by $\bar{v}_{e}$ measurements.

We examined this aspect of the summation method [10]. The calculated energy spectrum showed an unexpected level of consistency with measurements. This is particularly surprising given that we neglected the very significant, but difficult to quantify, systematic uncertainties discussed in the previous section. Fig. 2 shows the results of this calculation where the uncertainty band on the summation calculation only includes the uncertainties quantified within the ENDF nuclear database, and so should be considered as a lower bound on the $1 \sigma$ uncertainty. In this figure, all models and data are shown in ratio to a smooth approximation to the spectrum $F^{1}$ in order to highlight the details of the spectral shape.

$$
{ }^{1} F(E)=\exp \left(\sum_{i} \alpha_{i} E^{i-1}\right) \text { with } \alpha=\{0.4739,0.3877,-0.3619,0.04972,-0.002991\} .
$$




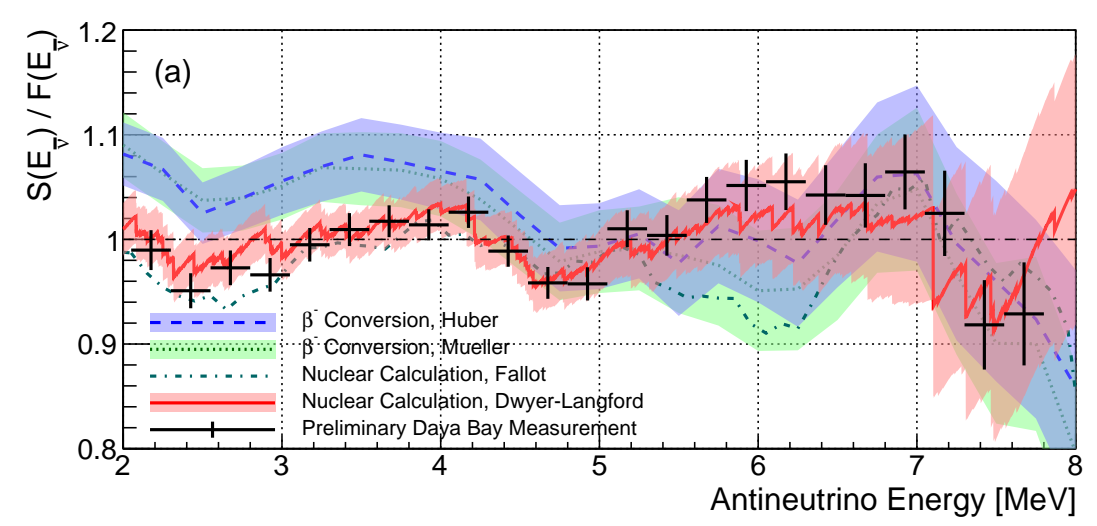

Figure 2: A detailed comparison of models of the $\bar{v}_{e}$ energy spectrum emitted by a nominal pressurized nuclear reactor. To highlight the differences between the models, all are shown in ratio to a smooth approximation to the spectrum, $F(E)$. The summation calculation using the ENDF/B.VII.i nuclear database (red solid line [10]) predicts a less steep spectrum and a shoulder between 5-7 MeV relative to the $\beta$-conversion models (blue dashed line [23], green dotted line [22]). While the shaded bands for the $\beta$-conversion models represent their actual $1 \sigma$ uncertainties, for the summation model the band is an underestimate since it only includes those uncertainties tabulated within the database. An alternate summation model (dashed-dotted line [27]), which relies on the JEFF nuclear database and a variety of measurements and theoretical models for characterizing nuclide decays, is consistent below $5 \mathrm{MeV}$ but deviates above this energy. The preliminary measurement of the $\bar{v}_{e}$ spectrum from the Daya Bay Experiment (black points [25]) is unexpectedly consistent with the ENDF/B.VII.i summation model, given the significant uncertainties in this calculation.

The summation method allowed us to dissect the calculated energy spectrum in the 5-7 MeV region in order to determine a possible origin for the shoulder. This calculation attributes $42 \%$ of the rate in this region to eight prominent beta decay transitions, which together produce a prominent shoulder. No other individual transition contributes more than $2 \%$ to this region, and collectively these low-rate transitions produce a residual smooth contribution to the total shape. Fig. 3 shows the calculated spectra from these prominent branches weighted by interaction cross-section, demonstrating how they produce a localized distortion of the spectrum in the 5-7 MeV region.

The summation method also highlights a commonly overlooked feature of reactor $\bar{v}_{e}$ spectra. The Coulomb field of the nucleus enhances the emission of low-energy electrons in beta decay. This simultaneously enhances the emission of high-energy $\bar{v}_{e}$, producing a kink or edge at the endpoint of the $\bar{v}_{e}$ energy spectrum for each transition (as can be seen in Fig. 3). These kinks introduce significant fine structure to the energy spectrum calculated by the summation method, as shown in Fig. 4. This structure is not currently visible due to the limited energy resolution, $\sim 8 \% / \sqrt{E[\mathrm{MeV}]}$, of the current generation of reactor $\bar{v}_{e}$ experiments. Future measurements of this spectral structure with resolution of $1 \%$ or better would open a unique and powerful window to the processes occurring within operating nuclear reactors. At the same time this structure could hinder future measurements which rely on accurate knowledge of the absolute energy spectrum, such as determination of the neutrino mass ordering $[7,8]$. 


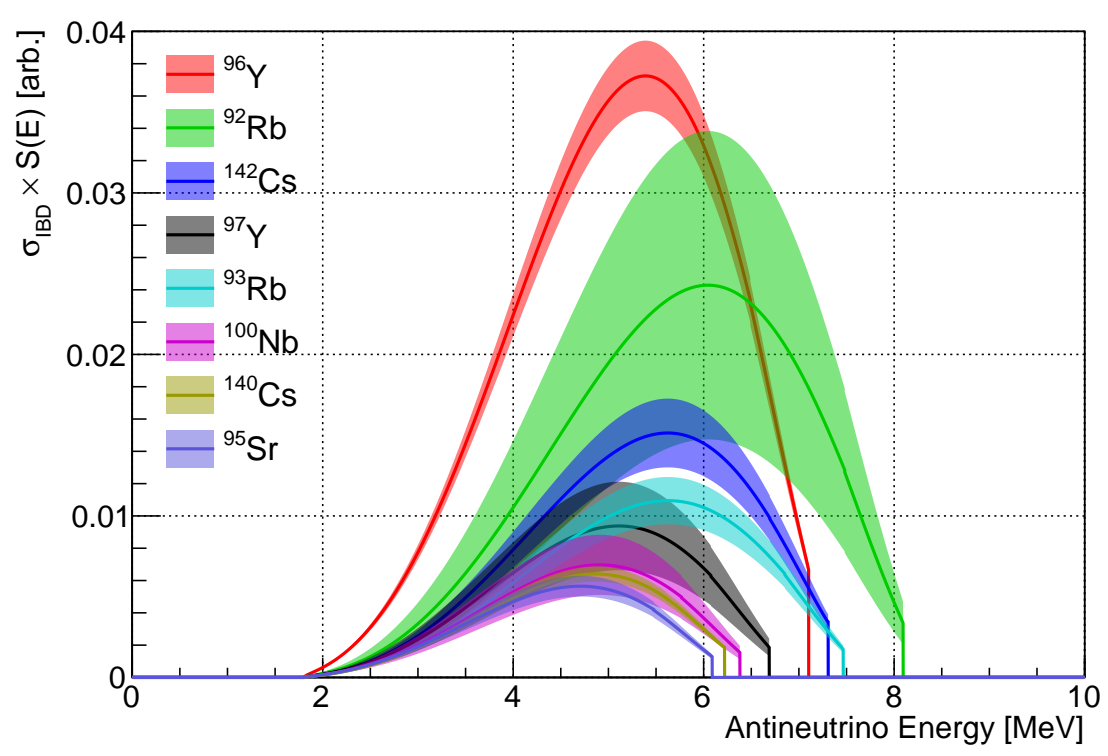

Figure 3: Antineutrino energy spectra weighted by the inverse beta decay interaction cross section for the eight decay transitions most prominent in the 5-7 MeV region of the shoulder. According to the ENDF/B.VII.i database, these eight ground-state to ground-state transitions contribute $42 \%$ of the expected rate at these energies, and in combination produce the recently observed spectral shoulder. The shaded bands represent the $1 \sigma$ uncertainties in their contributions according to their tabulated uncertainties in fission yields and relative decay probabilities. Recent total absorption gamma spectroscopy measurements [28] suggest the ${ }^{92} \mathrm{Rb}$ contribution is at the high end of the rather large uncertainty shown here, further enhancing the prediction of a shoulder.

\section{Discussion}

Are these eight prominent decay branches the correct explanation of the spectral deviation? Given the very significant uncertainties in the summation method, it is difficult to be conclusive. At the same time, it is rather suggestive that this summation calculation predicts a spectrum so similar to measurement. Since this calculation attributes the shoulder to only eight decay transitions, confirming the fission yields and relative probabilities for these decays would strongly substantiate the case.

On this topic, a body of nuclear structure measurements exist which have not yet been incorporated into the publicly-available nuclear databases [28, 29, 30, 31]. Recent work to incorporate these measurements suggest corrections which both enhance and diminish the $\bar{v}_{e}$ spectrum above $6.5 \mathrm{MeV}$. So far, these corrections do not dramatically alter the calculated shoulder. On the other hand, differences in tabulated fission yields between the ENDF and JEFF databases are pronounced [16]. The former predicts a shoulder as seen in the three recent $\bar{v}_{e}$ spectral measurements, while the latter predicts no shoulder, as seen in the ILL electron spectrum measurements.

Regardless of choice of nuclear database, the summation calculations either predict a shoulder for both the electron and $\bar{v}_{e}$ spectra, or for neither, due to the strong kinematic relationship between the electron and $\bar{v}_{e}$ in beta decay. This suggests a fundamental incompatibility between 


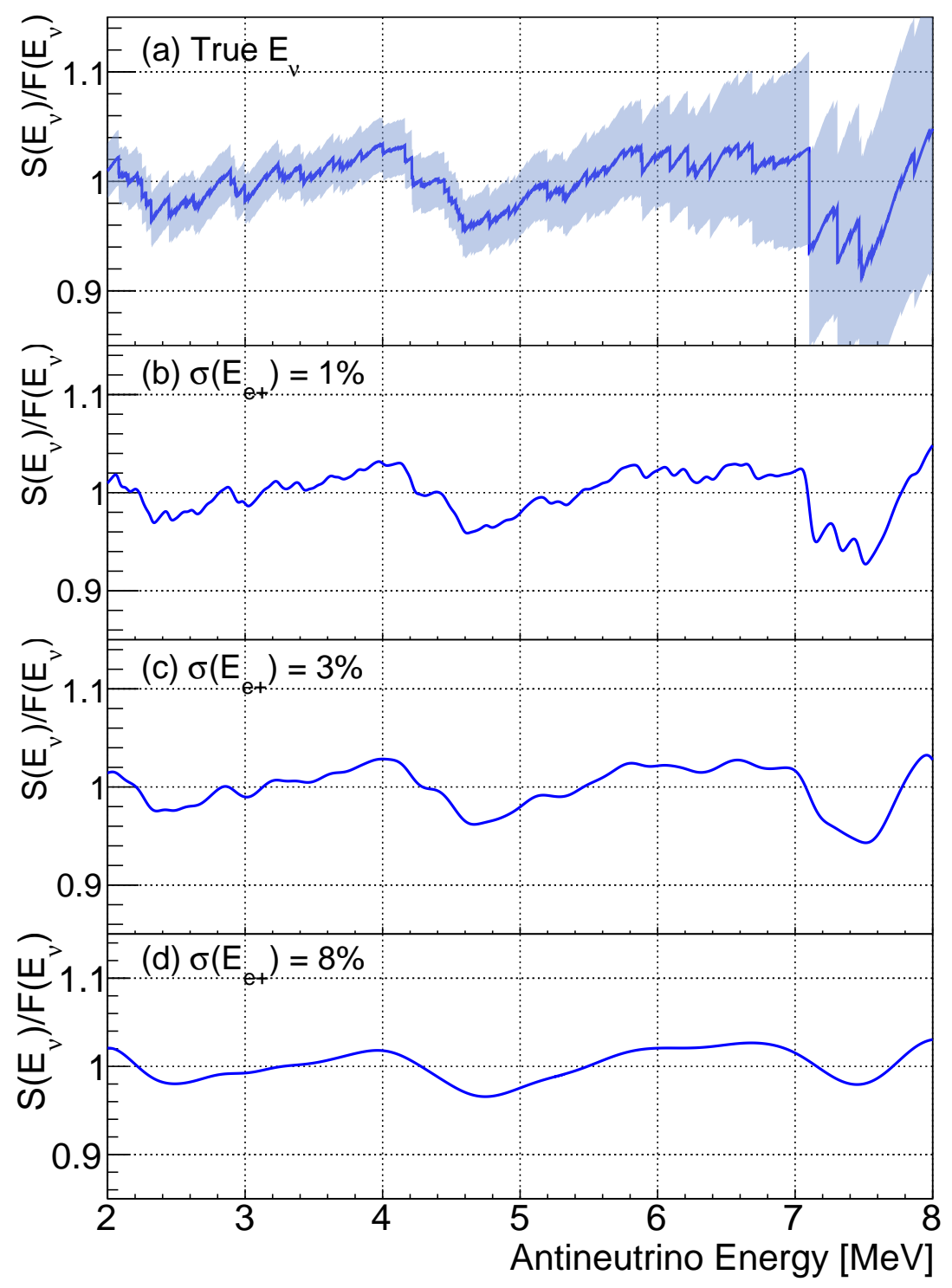

Figure 4: (a) The summation model predicts significant discontinuities in the reactor $\bar{v}_{e}$ spectrum due to nuclear Coulomb corrections. Each discontinuity is attributable to a single prominent beta decay transition occurring within the reactor. (b) Measurement using a detector with positron energy resolution of $1 \% / \sqrt{E[\mathrm{MeV}]}$ or better would reveal significant structure, providing a unique method to assess the physical processes occurring within a operating reactor. (c) This spectral structure may hinder future experiments which rely on knowledge of the spectral shape, such as determination of the neutrino mass ordering using a detector with $3 \%$ resolution. (d) Current measurements with $\sim 8 \%$ resolution are only able to reveal the broader spectral features. 
the recent reactor $\bar{v}_{e}$ measurements and the older ILL electron spectrum measurements. While an unforeseen systematic bias in these electron measurements could be to blame, it may still be possible to reconcile them with the recent $\bar{v}_{e}$ measurements. Differences in actinide fission rates or neutron energy spectra between the $\bar{v}_{e}$ and electron measurements could produce a relative difference in the fission yields for these two cases, so are difficult to rule out as possible origins [16]. Improved measurements of fission yields would help to discriminate these hypotheses [32, 33]. Measurements of the electron spectra for the most relevant decay transitions would significantly reduce uncertainties in $\bar{v}_{e}$ spectral shape [34]. Precision $\bar{v}_{e}$ spectral measurements at reactors with different actinide fission rates or neutron energy spectra would provide invaluable guidance on the origin of these discrepancies, and simultaneously allow direct tests of the sterile neutrino hypothesis [35]. Leveraging these same experiments to measure $\bar{v}_{e}$ spectra at high-resolution would also provide exceptional data for our understanding of nuclear reactors.

\section{Acknowledgments}

I would like to thank the organizers of the XVI International Workshop on Neutrino Telescopes for providing the opportunity to present this material, and for hosting a stimulating workshop. Thanks also go to Tom Langford for his contributions to the research discussed here. This work was supported under DOE OHEP DE-AC02-05CH11231.

\section{References}

[1] F. Reines, C. L. Cowan, F. B. Harrison, A. D. McGuire, and H. W. Kruse, Detection of the free anti-neutrino, Phys. Rev. 117 (1960) 159.

[2] T. Araki et al. (KamLAND Collaboration), Measurement of neutrino oscillation with KamLAND: Evidence of spectral distortion, Phys. Rev. Lett. 94 (2005) 081801, [hep-ex/ 0406035 ] .

[3] S. Abe et al. (KamLAND Collaboration), Precision Measurement of Neutrino Oscillation Parameters with KamLAND, Phys. Rev. Lett. 100 (2008) 221803, [arXiv:0801.4589] .

[4] F. P. An et al. (Daya Bay Collaboration), Spectral measurement of electron antineutrino oscillation amplitude and frequency at Daya Bay, Phys. Rev. Lett. 112 (2014) 061801, [arXiv: 1310.6732 ] .

[5] S.-H. Seo et al. (RENO Collaboration), New Results from RENO, , in proceedings of XV International Workshop on Neutrino Telescopes PoS (Neutel 2013) 018 (2013), [arXiv:1312.4111].

[6] Y. Abe et al. (Double Chooz Collaboration), Improved measurements of the neutrino mixing angle $\theta_{13}$ with the Double Chooz detector, JHEP 10 (2014) 086, [arXiv:1406. 7763 ] .

[7] Y. Wang, JUNO: A Multi-Purpose LS-based Experiment, in proceedings of XV International Workshop on Neutrino Telescopes POS (Neutel 2013) 030 (2013).

[8] S.-B. Kim, Proposal for RENO-50, in proceedings of International Workshop on RENO-50 toward Neutrino Mass Hierarchy (2013).

[9] G. Mention, M. Fechner, T. Lasserre, T. A. Mueller, D. Lhuillier, et al. The Reactor Antineutrino Anomaly, Phys. Rev. D83 (2011) 073006, [arXiv:1101.2755].

[10] D. A. Dwyer and T. J. Langford, Spectral Structure of Electron Antineutrinos from Nuclear Reactors, Phys. Rev. Lett. 114 (2015) 012502, [arXiv: 1407.1281 ] . 
[11] A. A. Sonzogni, T. D. Johnson, E. A. McCutchan, Nuclear structure insights into reactor antineutrino spectra, Phys. Rev. C91 (2015) 011301.

[12] T. R. England and B. F. Rider, Evaluation and compilation of fission product yields, ENDF 349 (1992).

[13] M. B. Chadwick et al., ENDF/B-VII.1 Nuclear Data for Science and Technology: Cross Sections, Covariances, Fission Product Yields and Decay Data, Nucl. Data. Sheets 112 (2011) 2887.

[14] J. K. Tuli, Evaluated nuclear structure data file, Nucl. Inst. and Meth. A369 (1996) 506.

[15] K.-H. Schmidt and C. Amouroux, General Description of Fission Observables, JEFF Report 24 (2014).

[16] A C. Hayes, J. L. Friar, G. T. Garvey, D. Ibeling, G. Jungman, et al., The Possible Origin and Implications of the Shoulder in Reactor Neutrino Spectra, arXiv: 1506.00583.

[17] A. C. Hayes, J. L. Friar, G. T. Garvey, G. Jungman, and G. Jonkmans, Systematic Uncertainties in the Analysis of the Reactor Neutrino Anomaly, Phys. Rev. Lett. 112 (2014) 202501, [arXiv:1309.4146].

[18] K. Schreckenbach, G. Colvin, W. Gelletly, and F. Von Feilitzsch, Determination of the anti-neutrino spectrum from U-235 thermal neutron fission products up to 9.5-MeV, Phys. Lett. B160 (1985) 325.

[19] F. Von Feilitzsch, A. A. Hahn, and K. Schreckenbach, Experimental beta spectra from Pu-239 and U-235 thermal neutron fission products and their correlated anti-neutrinos spectra, Phys. Lett. $\mathbf{B 1 1 8}$ (1982) 162.

[20] A. A. Hahn, K. Schreckenbach, W. Gelletly, F. von Feilitzsch, G. Colvin, et al., Anti-neutrino Spectra From ${ }^{241}$ Pu and ${ }^{239}$ Pu Thermal Neutron Fission Products, Phys. Lett. B218 (1989) 365.

[21] N. Haag, A. Gütlein, M. Hofmann, L. Oberauer, W. Potzel, et al. Experimental Determination of the Antineutrino Spectrum of the Fission Products of ${ }^{238}$ U, Phys. Rev. Lett. 112 (2014) 122501, [arXiv:1312.5601].

[22] T. A. Mueller, D. Lhuillier, M. Fallot, A. Letourneau, S. Cormon, et al., Improved Predictions of Reactor Antineutrino Spectra, Phys. Rev. C83 (2011) 054615, [arXiv:1101.2663] .

[23] P. Huber, On the determination of anti-neutrino spectra from nuclear reactors, Phys. Rev. C84 (2011) 024617, [arXiv:1106.0687].

[24] S.-H. Seo et al. (RENO Collaboration), New results from RENO, in proceedings of XXVIth International Conference on Neutrino Physics and Astrophysics (2014).

[25] F. P. An et al. (Daya Bay Collaboration), Measurement of the reactor antineutrino flux and spectrum at Daya Bay, arXiv: 1508.04233.

[26] J. C. Hardy, L. C. Carraz, B. Jonson, and P. G. Hansen, The essential decay of pandemonium: A demonstration of errors in complex beta-decay schemes, Phys. Lett. B71 (1977) 307.

[27] M. Fallot, S. Cormon, M. Estienne, A. Algora, V. M. Bui, et al., New antineutrino energy spectra predictions from the summation of beta decay branches of the fission products, Phys. Rev. Lett. 109 (2012) 202504, [arXiv:1208.3877].

[28] A.-A. Zakari-Issoufou, M. Fallot, A. Porta, A. Algora, J. L. Tain, et al., Total absorption spectroscopy study of ${ }^{92}$ Rb decay: a major contributor to reactor antineutrino flux, arXiv : 1504.05812 . 
[29] R. C. Greenwood, R. G. Helmer, M. A. Lee, M. H. Putnam, M. A. Oates, et al., Total absorption gamma-ray spectrometer for measurement of beta-decay intensity distributions for fission product radionuclides, Nucl. Instrum. Meth. A314 (1992) 514.

[30] O. Tengblad, G. Nyman, K. Aleklett, E. Lund, G. Rudstam, et al., Integral Anti-neutrino Spectra Derived From Experimental Beta Spectra of Individual Fission Products, Nucl. Phys. A503 (1989) 136.

[31] M. Wolinska-Cichocka, K. P. Rykaczewski, A. Fijalkowska M. Karny, R. K. Grzywacz, et al., Modular Total Absorption Spectrometer at the HRIBF (ORNL, Oak Ridge), Nucl. Data Sheets 120 (2014) 22.

[32] F. Martin, C. Sage, G. Kessedjian, C. O. Bacri, A. Bidaud, et al., Measurements of the Mass and Isotopic Yields of the ${ }^{233} U\left(n_{t h}, f\right)$ Reaction at the Lohengrin Spectrometer, Proceedings of the 2nd International Conference on Advancements in Nuclear Instrumentation Measurement Methods and their Applications (ANIMMA) (2011) 1.

[33] J. Ruz, D. M. Asner, R. G. Baker, J. Bundgaard, E. Burgett, et al., The NIFFTE project, arXiv:1309.7938.

[34] G. Li, R. Segel, N. D. Scielzo, P. F. Bertone, F. Buchinger, et al., Tensor Interaction Limit Derived From the $\alpha-\beta-\bar{v}$-Correlation in Trapped ${ }^{8}$ Li Ions, Phys. Rev. Lett. 110 (2013) 092502.

[35] J. Ashenfelter, A. B. Balantekin, H. R. Band, G. Barclay, C. Bass, et al., PROSPECT - A Precision Reactor Oscillation and Spectrum Experiment at Short Baselines, arXiv:1309.7647. 